EPJ Web of Conferences 61, 06008 (2013)

DOI: $10.1051 /$ epjconf/ 20136106008

(C) Owned by the authors, published by EDP Sciences, 2013

\title{
Evidence of Two-Component Optical Polarization in Blazar 1ES 1959+650
}

\author{
Marco Sorcia $^{1, a}$, Erika Benítez ${ }^{1, b}$, and David Hiriart ${ }^{2, c}$ \\ ${ }^{1}$ Instituto de Astronomía, Universidad Nacional Autónoma de México, AP 70-264, 04510 DF, Mexico \\ ${ }^{2}$ Instituto de Astronomía, Universidad Nacional Autónoma de México, AP 877, 22800, Ensenada BC, Mexico
}

\begin{abstract}
In this work we present an analysis of the optical polarimetric variability of the TeV-blazar 1 ES $1959+650$ from 2007 October 18 to 2011 May 5. The source showed a maximum variation of $1.2 \mathrm{mag}$ in $R$ band, and a maximum polarization degree of $12.2 \%$. The blazar presented a preferential position angle of the optical polarization of $\sim 153^{\circ}$, with variations of $10^{\circ}-50^{\circ}$, which is in agreement with the projected position angle of the parsec-scale jet found at $43 \mathrm{GHz}$. We infer the existence of the two optically thin synchrotron components that contribute to the polarized flux, consistent with the spine-sheath model. One of them is stable with a constant polarization degree of $4 \%$. Assuming a stationary shock, we estimated some parameters associated with the physics of the relativistic jet: the Doppler factor of $\delta_{D} \sim 23$, the viewing angle of the jet of $\Phi \sim 2^{\circ} .4$, and the size of the emission region of $r_{b} \sim 5.6 \times 10^{17} \mathrm{~cm}$.
\end{abstract}

\section{Introduction}

The TeV-blazar 1ES $1959+650$ was discovered in the radio band as part of a $4.85 \mathrm{GHz}$ survey performed with the $91 \mathrm{~m}$ NRAO Green Bank telescope [1]. Polarimetric observations at $43 \mathrm{GHz}$ VLBA from 2005-2009 revealed spine-sheath structures in the electric vector position angle (EVPA) [2]. These authors concluded that this blazar consists of a $\sim 1$ mas jet extending to the southeast at a position angle of about $150^{\circ}$. Furthermore, the source was observed also in the optical bands where it displayed large and fast flux variations [3]. The source is known to be hosted by an elliptical galaxy at $z=0.047$ and with $M_{R}=-23$. The spectral energy distrubution shows its first synchrotron peak at UV-X-ray frequencies. This object is classified as high-peaked BL Lac object (HBL). The first $\gamma$-ray signal at very high energies was reported in 1998 by the Seven Telescope Array in Utah [4]. In 2002 this object showed two TeV flares without simultaneous X-ray flares, a behavior sometimes referred to as orphan flares [3]. This blazar is listed in The First Catalog of AGN detected with Fermi Large Area Telescope [5]. In this work we report the results from the photopolarimetric monitoring of the TeV-blazar 1ES 1959+650. Our main goal is to establish the long-term optical variability properties of the polarized emission in the $R$-band. Variability of the Stoke's parameters obtained from our observations is analyzed in terms of a two-component model. Estimations of some physical parameters that are known to be associated with the kinematics of the relativistic jet were obtained. Also, we present a comparison of the polarization prop-

\footnotetext{
a e-mail: msorcia@astro.unam.mx

be-mail: erika@astro.unam.mx

ce-mail: hiriart@astrosen.unam.mx
}

erties found in our study with recent radio maps obtained with the VLBI at 43-GHz on this source by [2]. Our work suggests that the observed radiation originates in a region inside the jet where a standing shock is produced.

\section{Observations}

The observations were carried out with the $0.84 \mathrm{~m}$ f/15 Ritchey-Chrétien telescope at the Observatorio Astronómico Nacional of San Pedro Mártir (OAN-SPM) in Baja California, México. We used the instrument Polima which is a direct image polarimeter [see 6]. All observations were made as a part of a support program dedicated to the optical $R$-band monitoring of 37 blazars in the framework of the GLAST-AGILE Support Program (GASP) of the Whole Earth Blazar Telescope (WEBT). ${ }^{1}$ Polima consists of a rotating Glan-Taylor prism driven by a stepper motor with an accuracy of $0^{\circ} .1$. We used CCD's with pixel sizes go from 13.5 to $24 \mu \mathrm{m}$ and plate scales from 0.22 to 0.39 arc-sec pixel $^{-1}$. The exposure time was $240 \mathrm{~s}$ per image. The optical polarimetric monitoring was carried out from 2007 October $18 \mathrm{JD}(2454392)$ to 2011 May $5 \mathrm{JD}$ (2455687). During this period, we carried out 25 observing runs of seven nights per run, centered on the new moon phase when the object was visible. In total we collected 106 data points. Four images with the $R$-band filter and relative position angles were taken. The sequence of $0^{\circ}, 90^{\circ}, 45^{\circ}$, and $135^{\circ}$ for the relative prism position angle was used to reduce the sky variations influence to calculate both linear polarization and flux from the object. Data were reduced with a pipeline especially written for

\footnotetext{
${ }^{1}$ A detailed description of our photopolarimetric monitoring program on TeV Blazars can be found in http://www.astrossp.unam.mx/blazars
} 


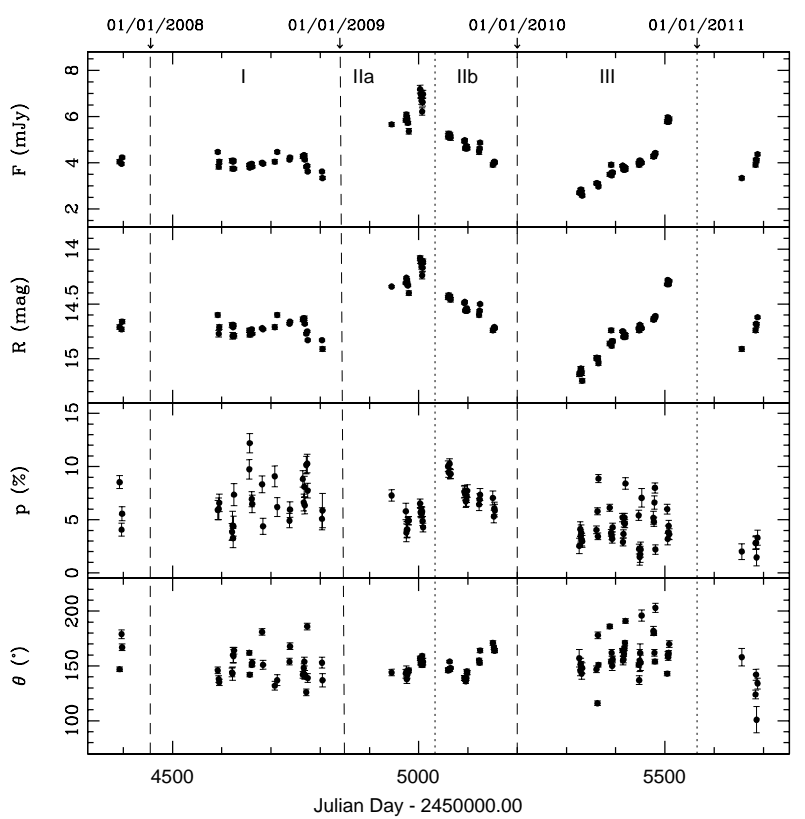

Figure 1. Light curves showing that the object displayed variations with timescales of $\sim$ days-months. From top to bottom: the $R$-band flux (mJy); the $R$-band (mag); the polarization degree $P(\%)$; and the polarization position angle $\theta\left({ }^{\circ}\right)$ vs Julian Date. Data are from 2007 October 18 to 2011 May 05. Each point has an associated error bar and corrected by the contribution of the host-galaxy

our monitoring program (Hiriart 2013, in prep). Polarimetric calibrations were done using the polarized standard stars ViCyg12 and HD155197 and the unpolarized standard stars GD319 and BD+332642 [7]. The magnitudes were measured using the aperture photometry technique. Then, we calculated the object's $R$-band magnitudes using comparison star 2, located $\sim 2$ '. 1 away from the target. The calibrated magnitude of the comparison star 2 in the $R$-band used is $12.53 \pm 0.02 \mathrm{mag}$, from [8].

\section{Results}

The $R$-band magnitudes were converted into apparent fluxes using the expression: $F_{\text {obs }}=K_{0} \times 10^{-0.4 m_{R}}$, with $K_{0}=3.08 \times 10^{6} \mathrm{mJy}$. The fluxes were corrected for the contribution of the host galaxy according to [9]. We have to consider the ambiguity of $180^{\circ}$ in the polarization angle. For this purpose we corrected the polarization angle assuming that the differences between the polarization angle of temporal adjacent data should be less than $90^{\circ}$. Figure 1 shows the $R$-band flux, the $R$-band light curve in magnitudes, the polarization degree $p$ and the position angle $\theta$ obtained for all 25 observing runs in $\sim 3$.6yr. For clarity, the entire period of observations has been divided into three main cycles: Cycle I: from 2008 May 04 to 2008 Dec 03. Cycle II: from 2009 Apr 22 to 2009 Nov 18. Cycle III: from 2010 May 08 to 2011 May 05.

From these data, a maximum of $R=14.08 \mathrm{mag}$ is observed, and a variation of $\Delta m_{R}=1.12 \mathrm{mag}$ in $\Delta t=329$

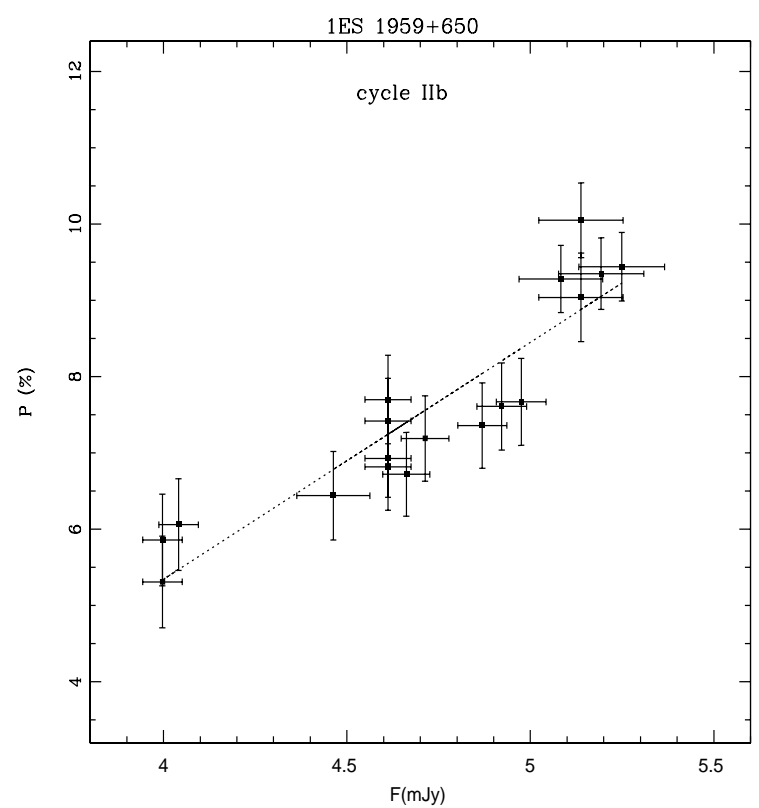

Figure 2. Correlation between the $R$-band flux and polarization degree from 2009 August 14 to November 18.

days is found. The peak flux of the object was $7.19 \pm 0.17$ mJy at 2009 June 19 . To establish a possible correlation between the polarization degree and the $R$-band fluxes, a Pearson's correlation coefficient was calculated $\left(r_{\text {pol }}\right)$. For Cycle IIb we found a strong correlation between these two parameters, with a Pearson's correlation coefficient of $r_{\mathrm{pol}}=0.984 \pm 0.025$ (see Figure 2 ). The polarization angle shows a general trend to remain around an average value of $153^{\circ} \pm 16^{\circ}$, with maximum variations of $\sim 10^{\circ}-50^{\circ}$ (see Figure 3). The minimum flux variability timescale obtained from our data in the $R$-band is $t_{\mathrm{var}}=9.74 \pm 1.17$ days.

To identify the presence of a stable polarized component, we define the average values of $Q$ and $U$ as the stationary polarization component. Figure 4 shows the $Q-U$ plane for the stable component. These average values appear as a black-filled star in the $Q-U$ plane plot, and correspond to a stable component with constant polarization degree $P_{c}=4.1 \% \pm 0.5 \%$ and polarization angle $\Theta_{c}=151^{\circ} \pm 13^{\circ}$. The constant polarization degree has a dispersion $\sigma_{P_{c}}=2.1 \%$. To estimate the variable component parameters, we looked for a possible linear relation between $Q$ vs $I$ and $U$ vs $I$ for the three relevant Cycles. For Cycle IIa (see Figure 5), our data show a linear tendency between these parameters. The polarization degree found for the variable component is $p_{\mathrm{var}}=$ $(46.8 \% \pm 4.9) \%$, with a polarization angle $\theta_{\mathrm{var}}=150^{\circ} \pm 4^{\circ}$.

\section{Discussion and conclusions}

Let us assume that the variable $R$-band flux component is produced by a standing shock, such that the source is a spherical blob of radius $r_{b}$ moving in a turbulent plasma, with a constant jet's Lorentz factor $\Gamma_{j}$. Thus, in the observer's reference frame, the flux of the shocked region is 


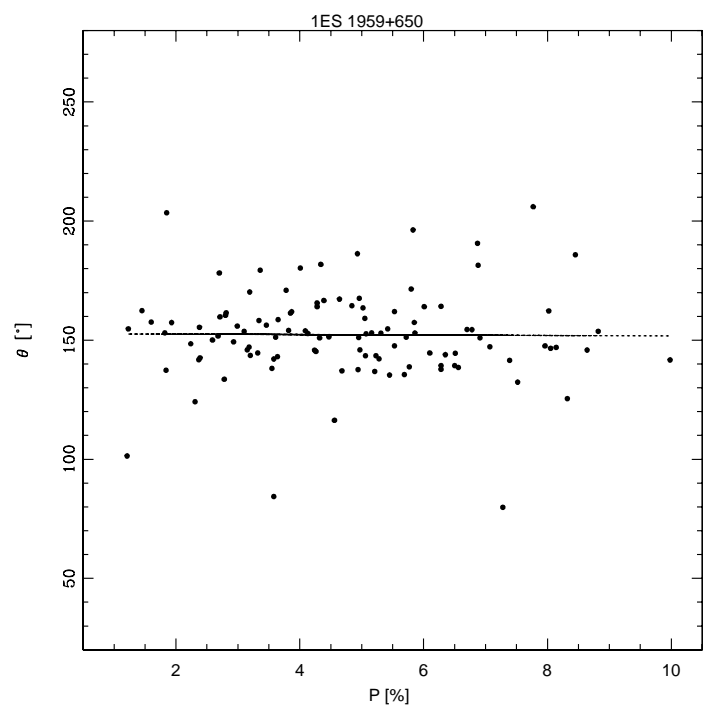

Figure 3. Correlation between the polarization angle and the polarization degree. There can be noticed a preferential tendency of the position angle $\sim 150^{\circ}$.

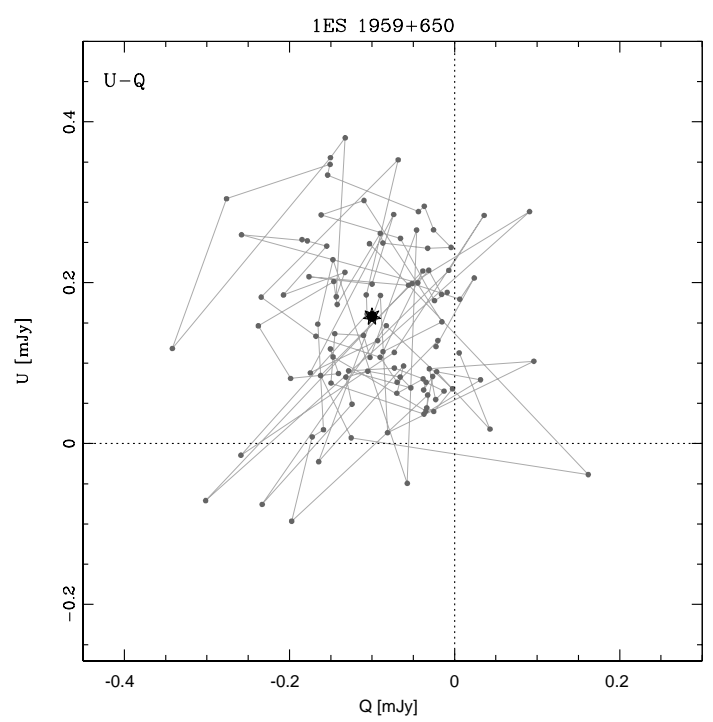

Figure 4. $Q-U$ absolute Stokes parameters plane obtained using all data. The solid black-filled star marks the mean constant value and the existence of a stable polarization component. The obtained average values of the absolute Stokes parameters $\langle Q\rangle$ and $\langle U\rangle$ are $-0.10 \pm 0.01 \mathrm{mJy}$ and $0.16 \pm 0.02 \mathrm{mJy}$, respectively. The polarization degree and position angle values obtained for the constant component are $P_{c}=(4.1 \pm 0.5) \%$ and $\Theta_{c}=151^{\circ} \pm 13^{\circ}$, respectively.

amplified as:

$$
F=F_{0} v^{-\alpha} \delta^{(3+\alpha)}
$$

where $\delta=\left[\Gamma_{j}(1-\beta \cos \Phi]^{-1}\right.$ is the jet's Doppler factor, $\beta=\left(1-\Gamma_{j}^{-2}\right)^{1 / 2}$ its global velocity in units of speed of light, and $\Phi$ is the viewing angle. The observed degree of polarization depends on the shock's viewing angle $\Psi$, the

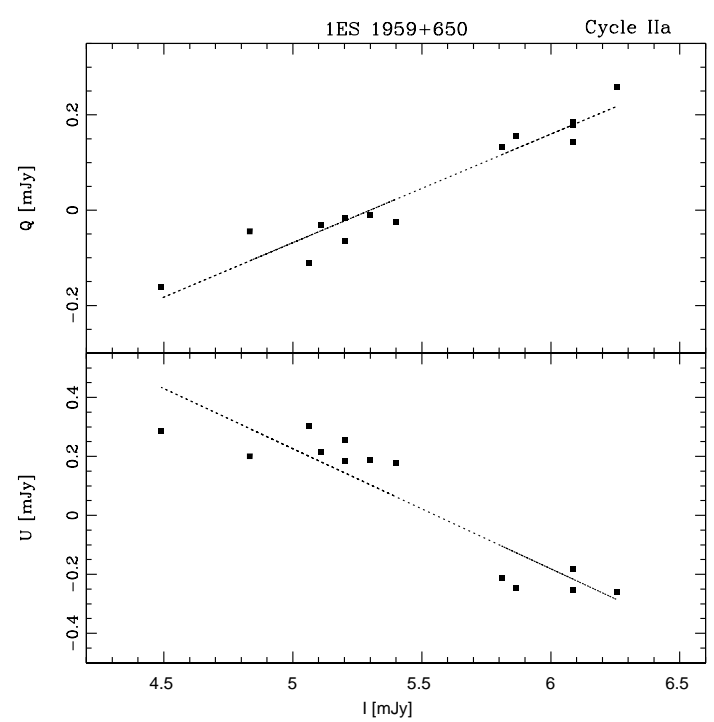

Figure 5. Linear correlation between the Stokes parameters $Q$ vs $I$ (top panel), and $U$ vs $I$ (bottom panel) for Cycle IIa (2009 AprJun). The correlation coefficients obtained for these parameters are $r_{q}=0.913$ and $r_{u}=-0.902$, and the slopes $m_{q}=0.228 \pm 0.028$, $m_{u}=-0.408 \pm 0.054$, respectively.

spectral index $\alpha$, and the ratio of densities of the shocked region to the unshocked region $\eta=\eta_{\text {shock }} / \eta_{\text {unshock }}[10]$ :

$$
p \approx \frac{\alpha+1}{\alpha+5 / 3} \frac{\left(1-\eta^{-2}\right) \sin ^{2} \Psi}{2-\left(1-\eta^{-2}\right) \sin ^{2} \Psi},
$$

and

$$
\Psi=\tan ^{-1}\left\{\frac{\sin \Phi}{\Gamma_{j}\left(\cos \Phi-\sqrt{1-\Gamma_{j}^{-2}}\right)}\right\}
$$

We assumed a bulk Lorentz factor $\Gamma_{j}=18$, and value of $\alpha_{\mathrm{ox}}=1.64$, given by [11]. From these equations, we can estimate the Doppler factor as a function of time. We adopt $\eta=2.3$, which is the minimum possible compression that produces a degree of linear polarization as high as $47 \%$. From Figure 6, it can be seen that when the source shows its maximum brightness (14.08 mag, JD 2455002), the Doppler factor reaches $\delta \sim 23$, the viewing angle of the jet show a minimum value of $\Phi \sim 2^{\circ} .3$, and the viewing angle of the shock $\Psi \sim 73^{\circ}$ undergoes its maximum aberration due to relativistic effects. This finding may explain the high degree of polarization found in the low activity phase. That is, the amplification of the magnetic field components parallel to the shock due to Doppler effect is not enough to considerably increase the polarization degree. In this context, the shock could be affecting an emission zone with a magnetic field almost parallel to the jet axis. We find the maximum compression of the plasma $\eta=1.145$ when the polarization degree reaches its maximum value of $10.3 \%$ (JD 2455062). 


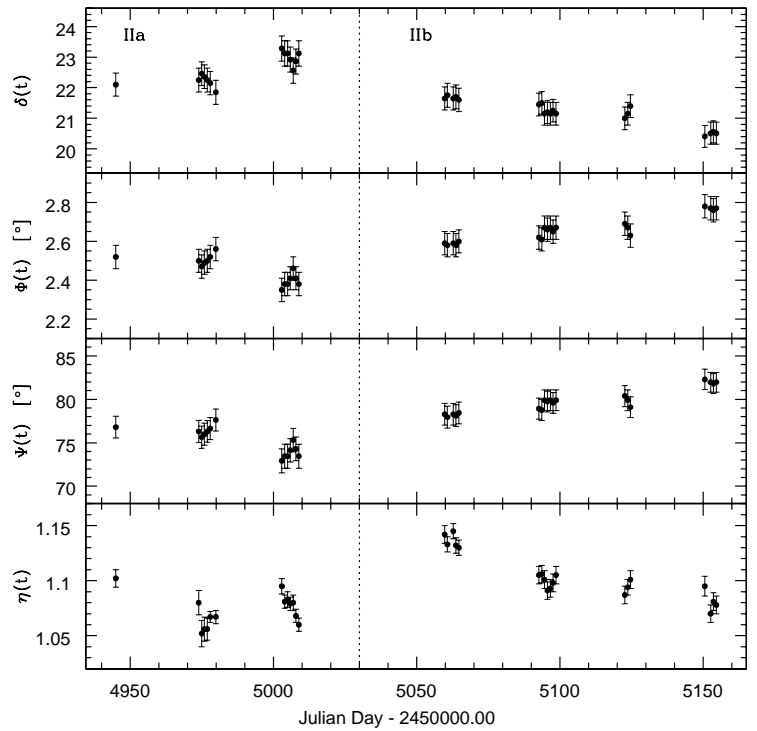

Figure 6. Temporal variability of some physical parameters related to the relativistic jet kinematics of 1ES 1959+650 derived in this work. From top to bottom panels: the Doppler factor, $\delta(t)$; the angle between the jet axis and the line of sight, $\Phi(t)$; viewing angle of the shock, $\Psi(t)$; and the compression factor of the shocked to the unshocked plasma, $\eta(t)$, during the high activity state in 2009.

On the other hand, the lifetime of the synchrotron electrons for a given frequency $v$ in $\mathrm{GHz}$ is [12]:

$$
t_{\mathrm{var}}=4.75 \times 10^{2}\left(\frac{1+z}{\delta_{0} v_{\mathrm{GHz}} B^{3}}\right)^{1 / 2} \text { days } .
$$

From our data [6] we find $t_{\mathrm{var}}=9.7 \pm 1.2$ days, and $\delta_{0}=23$. From here, we obtain the magnetic field intensity $B=0.061 \pm 0.005 \mathrm{G}$., and an upper limit to the emission region size of $r_{b} \leq c t_{\mathrm{var}} \delta_{0} /(1+z)=(5.61 \pm 0.68) \times 10^{17} \mathrm{~cm}$.

From photometric and polarimetric analysis we conclude that in general, there could not be found any correlation between the polarization degree and the optical flux, except when the source started to decrease its brightness after the first maximum occurred. The minimum variability timescale of the $R$-band flux was found to be $\sim 10$ days for the entire period of observations. The source presented a preferential average position angle of $153^{\circ} \pm 16^{\circ}$. This preferred position angle of the optical polarization is in good agreement with the projected position angle of the parsec scale jet found by [2] from $43 \mathrm{GHz}$ images. The analysis done with the Stokes parameters reveals the existence of two components that contribute to the polarized flux, such that the polarimetric behavior is consistent with a spine-sheath structure of the jet. Finally, the $R$-band observed variations can be explained in the framework of a standing-shock model.

\section{Acknowledgements}

M.S., E.B. and D.H. thank financial support from UNAMDGAPA-PAPIIT through grant IN116211-3. D.H. acknowledge financial support from CONACyT, Mexico, thru grant 106719. This research has made use of the SAO/NASA Astrophysics Data System (ADS) and of the NASA/IPAC Extragalactic Database (NED), which is operated by the Jet Propulsion Laboratory, California Institute of Technology, under contract with the National Aeronautics and Space Administration.

\section{References}

[1] R.H. Becker, R.L. White, A.L. Edwards, ApJS75, 1 (1991)

[2] B.G. Piner, N. Pant, P.G. Edwards, ApJ723, 1150 (2010), 1009.2269

[3] H. Krawczynski, S.B. Hughes, D. Horan, F. Aharonian, M.F. Aller, H. Aller, P. Boltwood, J. Buckley, P. Coppi, G. Fossati et al., ApJ601, 151 (2004), arXiv: astro-ph/0310158

[4] T. Nishiyama, "Detection of a new TeV gamma-ray source of BL Lac object 1ES 1959+650", in International Cosmic Ray Conference (1999), Vol. 3 of International Cosmic Ray Conference, p. 370

[5] A.A. Abdo, M. Ackermann, M. Ajello, et al., ApJ715, 429 (2010), 1002 . 0150

[6] M. Sorcia, E. Benítez, D. Hiriart, J.M. López, J.I. Cabrera, R. Mújica, J. Heidt, I. Agudo, K. Nilsson, M. Mommert, ApJS206, 11 (2013), 1304 . 2819

[7] G.D. Schmidt, R. Elston, O.L. Lupie, AJ104, 1563 (1992)

[8] M. Villata, C.M. Raiteri, L. Lanteri, G. Sobrito, M. Cavallone, A\&AS130, 305 (1998)

[9] K. Nilsson, M. Pasanen, L.O. Takalo, E. Lindfors, A. Berdyugin, S. Ciprini, J. Pforr, A\&A475, 199 (2007), 0709.2533

[10] P.A. Hughes, L. Miller, Introduction: synchrotron and inverse-Compton radiation (1991), p. 1

[11] A.A. Abdo, M. Ackermann, I. Agudo, M. Ajello, H.D. Aller, M.F. Aller, E. Angelakis, A.A. Arkharov, M. Axelsson, U. Bach et al., ApJ716, 30 (2010), 0912.2040

[12] V.A. Hagen-Thorn, V.M. Larionov, S.G. Jorstad, A.A. Arkharov, E.I. Hagen-Thorn, N.V. Efimova, L.V. Larionova, A.P. Marscher, ApJ672, 40 (2008), 0709.3550 